\title{
Afasia de conducción como consecuencia de un astrocitoma anaplásico parieto-temporo- occipital izquierdo: estudio de caso
}

\author{
Conduction Aphasia as a Result of Left Parietal-Temporal- \\ Occipital Anaplastic Astrocytoma: A Case Study
}

Recibido: septiembre 2 de 2010 | Revisado: diciembre 1 de 2010 | Aceptado: diciembre 16 de 2010

\author{
Oscar Mauricio Aguilar Mejía ** \\ BEATRIZ RAMírez BERMEJO \\ JuAN CARlos ACEVEdo GONZÁlez **** \\ Miguel EnRIQue Berbeo CALDERÓN \\ Pontificia Universidad Javeriana, Bogotá, Colombia
}

Para citar este artículo. Aguilar, O. M., Ramírez, B. Acevedo, J. C., \& Berbeo, M. E. (2011). Afasia de conducción como consecuencia de un astrocitoma anaplástico parieto-temporo-occipital izquierdo: estudio de caso. Universitas Psychologica, 10(1), 163-173.

* Artículo de investigación en neuropsicología.

** Departamento de Psicología, Cr 5 \# 39-00 piso 2, oficina 218. Bogotá, Colombia. E-mails: oaguilar@ javeriana.edu.co ; betty_bermejo@hotmail.com

**** Hospital Universitario San Ignacio, Departamento de Neurociencias, Cr. 7 \# 40-62 6o Piso. Bogotá, Colombia. E-mails: juan.acevedo@javeriana.edu. co ; miguel.berbeo@javeriana.edu.co

\section{RESUMEN}

La afasia de conducción es un trastorno caracterizado por un lenguaje espontáneo relativamente fluido, buena comprensión, pero dificultades en la repetición asociadas con parafasias fonológicas. Se ha atribuido a lesiones del fascículo arqueado por desconexión entre el lóbulo temporal posterior y el frontal; sin embargo, se ha debatido esta postura, planteando que la integridad y funcionamiento del fascículo arqueado no es indispensable en la repetición verbal. Se presenta un caso de un sujeto varón de 23 años que, como consecuencia de un astrocitoma anaplásico recidivante que abarca áreas parietales y temporo-occipitales, presenta una afasia de conducción. Se plantea una reconceptualización de esta afasia, analizándola en términos clínicos, neuropsicológicos y en las redes neuronales existentes entre áreas cerebrales posteriores ipsilaterales y contralaterales.

Palabras clave autores

Afasia de conducción, fascículo arqueado, repetición.

Palabras clave descriptores

Afasiología, lateralización del lenguaje, lesión neurológica.

\section{A B S T R A C T}

Conduction aphasia is a language disorder characterized by an impaired ability to repeat verbal material associated with phonological paraphasias but a relatively fluent spontaneous speech and preserved comprehension. It has been attributed to lesions of the arcuate fasciculus by disconnection between posterior temporal lobe and frontal lobe, however, this idea has been debated, because the integrity and function of the arcuate fasciculus does not seem to be essential in verbal repetition. We report a case of a 23 year old male, with conduction aphasia as a result of a recurrent anaplastic astrocytoma in parietal and temporo-occipital areas. We propose a reconceptualization of the aphasia, analyzing it in terms of clinical neuropsychological and neural networks between ipsilateral and contralateral posterior brain areas.

Key words authors

Conduction Aphasia, Arcuate Fasciculus, Repetition.

Key words plus

Aphasiology, Lenguage Lateralization, Neurological Lesion. 


\section{Introducción}

La afasia de conducción, descrita por Wernicke en 1874, es usualmente definida como un trastorno afásico caracterizado por un lenguaje espontáneo relativamente fluido, buena comprensión, pero dificultades en la repetición asociadas con abundantes parafasias fonológicas (Anderson et al., 1999; Baldo, Klostermann \& Dronkers, 2008; Bernal \& Ardila, 2009; Buchsbaum et al., 2011; Geschwind, 2009). A partir de los casos estudiados, Wernicke postuló que así como el área perisilviana anterior, descrita por Paul Broca, era importante en la producción del lenguaje y su alteración producía una afasia no fluente, la región cortical en la porción posterior del lóbulo temporal superior contenía los recuerdos de cómo suenan las palabras, y señaló que la causa etiológica de este desorden era la desconexión de estas dos áreas. Más adelante, en 1960, Geschwind planteó que la desconexión de estas dos áreas se daba cuando existía una lesión o interrupción del fascículo arqueado (Anderson et al., 1999; Geschwind, 2009).

Los pacientes con afasia de conducción también pueden presentar dificultades en la nominación, en la lectura y en la escritura, así como alteraciones primarias tales como hemiparesia derecha y pérdida sensorial cortical (Bernal \& Ardila, 2009). Son frecuentes, en los pacientes con afasia de conducción, los intentos por corregir sus errores de pronunciación, lo que ha recibido el nombre de "conduite d'approche" (Bartha, Mariën, Powe \& Benke, 2004; Buchsbaum et al., 2011).

Algunos autores se han preguntado si la afasia de conducción es un diagnóstico topográfico o sindromático, pues si se trata de este último, muchos casos podrían incluirse bajo el mismo diagnóstico, incluso en pacientes con lesiones corticales. En estos casos, podría encontrarse anomia, agrafia y otros trastornos del lenguaje, dependiendo de la extensión de la lesión cortical. Por otro lado, si fuera únicamente un síndrome de desconexión, se esperaría encontrar un cuadro más limitado, probablemente con déficits fonológicos, correlacionados con interrupciones funcionales del fascículo arqueado producidos por estimulación eléctrica intraoperatoria (Bernal \& Ardila, 2009). Además, y como explicaciones más distantes, diversos autores han planteado que la afasia de conducción se puede explicar por déficits en la memoria a corto plazo (Baldo, Klostermann \& Dronkers, 2008) y como una forma de apraxia ideomotora (Robin, Jacks, Hageman, Clark \& Woodworth, 2008).

Como se mencionó anteriormente, la afasia de conducción ha sido tradicionalmente explicada como un síndrome de desconexión entre las áreas de Broca y Wernicke, debido a una lesión que afecta el fascículo arqueado; sin embargo, es importante resaltar que los casos reportados de afasia de conducción normalmente son causados por infartos y tumores, los cuales afectan, directa o indirectamente, otras áreas adyacentes, incluso la corteza cerebral. En efecto, es extremadamente improbable encontrar un caso de afasia de conducción con una lesión limitada al fascículo arqueado. Ciertamente, el fascículo arqueado ha sido reportado como involucrado en la afasia de conducción, pero lesiones corticales por sí solas, sin extensión subcortical, también pueden producir el mismo cuadro afásico; así mismo, y contrario a la semiología clínica clásica, se han reportado casos en los que pacientes con lesiones del fascículo arqueado pueden preservar la habilidad de repetir. De todo lo anterior, se podría concluir que el fascículo arqueado parece estar implicado funcionalmente en la repetición verbal, pero su integridad y su funcionamiento no son indispensables para tal proceso cognitivo (Anderson et al., 1999; Bernal \& Ardila, 2009; Buchsbaum et al., 2011).

Siendo la repetición verbal el déficit central del trastorno, se han estudiado los aspectos relacionados con dicha capacidad, mostrando que se deben tener en cuenta al menos dos subsistemas neurales, uno para la vocalización y otro para transferir las claves decodificadas en las áreas auditivas hacia las áreas de programación motora, representación y ejecución. El primero se relaciona con áreas motoras, premotoras y con el tronco encefálico (núcleos vocales) y el segundo, con el fascículo arqueado u otra vía de conexión similar. Este interjuego mecánico entre esquemas fonológicos y secuencias motoras, no está necesariamente ligado a la extracción 
semántica o a la conciencia semántica, ya que una persona puede repetir palabras, pseudopalabras e incluso frases en su lengua materna o en un idioma extranjero, sin entender el significado; esto implicaría que la repetición no debe ser considerada como un componente del lenguaje, sino del habla; por lo que la afasia de conducción podría ser interpretada como un desorden del habla y no del lenguaje, y por lo tanto no como una afasia, o al menos no como una "afasia primaria". Puede conjeturarse que cualquier sistema de repetición vocal, además, necesita un subsistema que lleve información desde las áreas auditivas receptivas a las áreas de ejecución. Este es el punto en el que el fascículo arqueado juega un papel crucial (Bernal \& Ardila, 2009).

Usualmente, en la literatura neurológica, la terminación rostral del fascículo arqueado ha sido asignada al área de Broca (Catani \& Mesulam, 2008). En contraste con la abundante literatura que acepta está conexión entre Broca y Wernicke, los nuevos hallazgos no apoyan este punto de vista. Bernal y Altman (2008 citados por Bernal \& Ardila, 2009) usando tractografía con tensor de difusión en un estudio con 12 sujetos diestros sin déficit neurológico, se centraron en analizar las fibras del fascículo arqueado, las cuales se obtuvieron bilateralmente mediante la ubicación de una región de interés en forma triangular lateral a cada uno de los tractos corticoespinales, en un plano coronal a lo largo del la cara rostral del esplenio del cuerpo calloso. Estos autores encontraron que las proyecciones del fascículo arqueado al área de Broca estaban ausentes en 10 de los sujetos (83.3 \%) y eran mínimas en 2 de los sujetos (16.6\%). Sin embargo, las terminaciones del fascículo arqueado fueron encontradas en el giro precentral en el 100 $\%$ de los casos. La conclusión general a la que llegaron, es que el fascículo arqueado no conecta con el área de Broca, en cambio, conecta con el giro precentral (áreas motora primaria) y con el área premotora. Teóricamente, la conexión rostral del fascículo arqueado con las áreas motora y premotora no necesariamente entra en conflicto con alguna regla neurológica o neuroanatómica establecida; en este tercio inferior, el giro precentral está divi- dido en dos partes: el banco anterior y parte del aspecto convexo del giro es la sede del área 6 de Brodmann, el resto del giro está representado por el área 4 de Brodmann, que corresponde a la representación somatotópica de la lengua, los labios y la faringe. Evidencia reciente sugiere que el área 6 de Brodmann está involucrada en la programación del habla y el procesamiento fonológico y léxico, por tanto, la participación de ésta en la programación del habla lo hace un blanco muy adecuado para el fascículo arqueado (Bernal \& Ardila, 2009).

Estudios electrocorticales han encontrado que el fascículo arqueado no solo transmite información desde las áreas temporales a las frontales, sino también en la dirección opuesta (Matsumoto et al., 2008). Esta observación sugiere que el papel del fascículo arqueado puede ser notablemente más complejo que la simple transmisión de información entre las áreas de Wernicke y Broca. Este flujo bidireccional de información parece apoyar la conexión del fascículo arqueado con las áreas premotoras, ya que cierta retroalimentación de la articulación puede ser necesaria (Liberman, Cooper, Shankweiler \& Studdert-Kennedy, 1967 citados por Bernal \& Ardila, 2009). Estas teorías sugieren que los mismos procesos involucrados en la producción, también participan en la percepción del lenguaje, por lo tanto, la transferencia bidireccional de la información sugiere que, en cierta medida, la información sobre la producción del lenguaje es importante para la comprensión del mismo. Diversos hallazgos parecen apuntar a una función accesoria del fascículo arqueado en la repetición del habla, y no una única, segregada y necesaria función, lo que da lugar a la posibilidad de que el fascículo arqueado podría funcionar como un complemento para facilitar la repetición o para monitorear el habla a nivel fonológico (Bernal \& Ardila, 2009).

Una conexión así sugiere que la representación auditiva del lenguaje juega un papel directo en la planificación motora verbal, y favorece la explicación dada por Luria (1976 citado por Bernal $\&$ Ardila, 2009) quien ve las dificultades en la repetición de la afasia de conducción como una apraxia ideomotora verbal. Además, facilita la explicación del fenómeno de conduite d'approche 
como una desconexión del área premotora. En efecto, una desconexión premotora podría impedir la secuenciación motora apropiada, necesaria para pronunciar los componentes de una palabra (sílabas) que, como resultado genera las parafasias fonológicas. Dado que las áreas exclusivas del lenguaje no se afectan, la conciencia fonológica tampoco lo hace, y el paciente intenta corregir sus errores de pronunciación exhibiendo la típica conduite d'approche, de la misma manera que un paciente apráxico intenta una y otra vez realizar los movimientos adecuados que le permitan ejecutar una acción específica. Esto contrasta con las parafasias fonológicas propias del lenguaje, en las cuales el paciente usualmente no es consciente de su error y, por lo tanto, no se dan estos intentos de autocorrección (Bernal \& Ardila, 2009).

\section{Descripción del Caso HJ}

Paciente varón de 23 años, diestro, con estudios en educación básica primaria que trabaja como fumigador en poblaciones cercanas a la ciudad de Bogotá. Como consecuencia de un astrocitoma anaplásico recidivante que abarca principalmente el lóbulo parietal izquierdo, pero que también compromete áreas temporo-occipitales (véase Figura 1), y por un síndrome convulsivo ingresa en julio de 2010 al servicio de neurocirugía del Hospital Universitario San Ignacio de la ciudad de Bogotá. Se solicita valoración neuropsicológica pre y posquirúrgica, pero adicionalmente se quería descartar un posible Síndrome de Gerstmann secundaria a la alteración parietal izquierda. Como antecedente quirúrgico relevante, al paciente dos meses atrás se le había realizado la primera resección del astrocitoma sin consecuencias en su lenguaje. Al momento de la evaluación neuropsicológica, la familia refiere como problema central las dificultades de $\mathrm{HJ}$ para pronunciar y para repetir palabras, al igual que manifiestan que no ha tenido cambios de comportamiento.

\section{Resonancia magnética nuclear}

Se realiza resonancia cerebral simple y con contraste. En cortes axiales, sagitales y coronales en
T1 (sin gadolinio) muestra una lesión de aspecto hipointensa localizada sobre los lóbulos parietal, temporal y occipital. Las aéreas de Broadman comprometidas son el área 7, 19, 39 y 40. También compromete el fascículo arqueado. En proyecciones en T1 con gadolinio muestra una lesión que realza de forma heterogénea con el medio de contraste, centro necrótico y un gran halo de edema vasogénico. Esta lesión se localiza sobre el aspecto posterior del lóbulo parietal izquierdo, temporal posterior y superior izquierdo y anterior del lóbulo occipital.

\section{Evaluación neuropsicológica}

A partir de la alteración neurológica de $\mathrm{HJ}$ y de la información obtenida en la entrevista inicial, se utiliza un protocolo de evaluación conformado por los subtests de orientación, lenguaje, memoria verbal, praxias y gnosias del Programa Integrado de Exploración Neuropsicológica Barcelona (PeñaCasanova, 1990).

\section{Descripción de los tests}

\section{Orientación}

Consta de 18 preguntas referidas a orientación en persona (7), en lugar (5) y en tiempo (6).

\section{Praxias}

- Orofonatorias en imitación: se compone de 10 ítems (20 puntos) en el que la persona debe imitar movimientos y sonidos con la boca.

- Gesto simbólico de comunicación: se trata de gestos simples, realizados con una sola extremidad y en la que no se implica ningún objeto. Se realiza a la orden (10) y a la imitación (10).

- Mímica del uso de objetos: se solicita al paciente que a la orden verbal y a la imitación, realice la mímica del uso de 5 objetos.

- Imitación de posturas unilateral: se compone de 5 ítems que se deben realizar con la extremidad superior derecha y luego con la extremidad superior izquierda.

- Praxis constructiva gráfica: con la mano do- 


\section{Figura 1A}

Resonancia cerebral en cortes axiales, coronales y sagitales, proyecciones en T1 simple (sin gadolinio) que muestra una lesión de aspecto hipointensa localizada sobre los lóbulos parietal, temporal y occipital. Las áreas de Brodmann comprometidas son el área 7, área 19, área 39 y área 40. Resonancia cerebral en cortes axiales, coronales y sagitales, en T1 con gadolinio que muestra una lesión que realza de forma heterogénea con el medio de contraste, centro necrótico y un gran halo de edema vasogénico. Esta lesión se localiza sobre el aspecto posterior del lóbulo parietal izquierdo, temporal posterior y superior izquierdo y anterior del lóbulo occipital. Las áreas de Brodmann comprometidas son el área 7, área 19, área 39 y área 40.
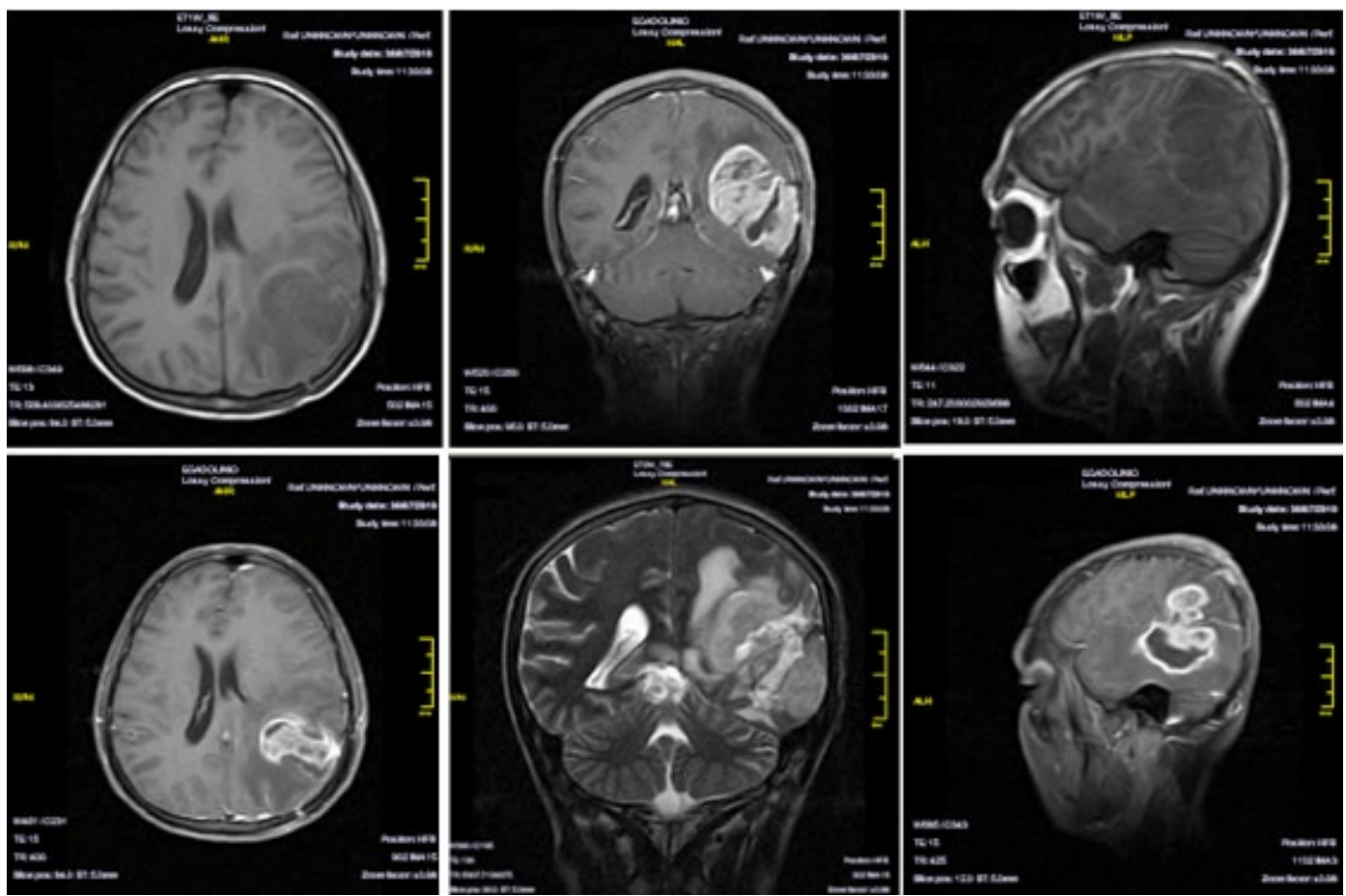

Fuente: elaboración propia.

minante se solicita la realización de figuras a la orden y a la copia.

\section{Lenguaje}

- Fluencia verbal y contenido informativo: se valora a partir de la conversación en la entrevista, de la narración acerca de un tema específico y de la descripción de una lámina.

- Prosodia: los datos se obtienen a partir del lenguaje espontáneo y del contacto verbal inicial.

- Lenguaje automático - Control mental: se estudia la capacidad de decir en orden directo e inverso, la serie numérica de 1 a 20, los días de la semana y los meses del año.
- Repetición: se compone de 6 partes en la que el paciente debe repetir silabas (8), pares de silabas (8), logatomos (8), pares de palabras (8), palabras (10) y frases (9).

- Denominación visuoverbal: se divide en tres partes. Denominación de 14 imágenes, de 6 objetos y 6 partes del cuerpo.

- Comprensión verbal: se realiza a partir de otorgar órdenes al paciente buscando que señale 12 imágenes (emparejamiento palabra-imagen), que señale 6 partes del cuerpo (emparejamiento palabra-parte del cuerpo), que realice actividades solicitadas a partir de 16 órdenes verbales, y que dé respuestas de "sî" o "no" a partir de material ideativo complejo (9 instrucciones). 


\section{Memoria verbal}

- Memoria verbal de textos: se explora mediante la repetición inmediata y diferida de dos textos (23 elementos). El estudio diferido se realiza a los 5 minutos después de haber finalizado el estudio inmediato.

\section{Escritura}

- Mecánica de la escritura: se estudia mediante la escritura del nombre, de la dirección del paciente y de la copia de una frase.

\section{Cálculo}

- Cálculo mental: se estudia mediante la realización de 2 sumas, 2 restas, 3 multiplicaciones y 3 divisiones simples.

- Cálculo escrito: se realizan 2 sumas, 2 restas, 1 multiplicación y 1 división.

\section{Gnosias Digítales}

- Reconocimiento digital: se compone de 3 partes. La identificación de dedos aislados con la mano visible (5 intentos); la identificación de dedos aislados con la mano no visible (5 intentos); y la identificación de pares de dedos tocados simultáneamente con la mano no visible (5 intentos);

\section{Orientación derecha-izquierda}

El test se compone de 10 ítems en el que el paciente debe señalar las partes laterales del cuerpo solicitadas por orden verbal (Peña-Casanova, 1990).

\section{Resultados}

En la evaluación prequirúrgica, HJ se encontraba alerta, orientado en persona, levemente desorientado en lugar y severamente desorientado en tiempo. Obtuvo desempeños adecuados en los subtests de escritura, cálculo, gnosias digitales y orientación derecha-izquierda, por lo cual se descartó un diagnóstico de síndrome de Gerstmann. Se evidenció una buena articulación y prosodia del lenguaje, pero con una fluidez moderadamente afectada (cantidad de palabras producidas), con abundantes parafasias fonológicas y con menor frecuencia semánticas, así como dificultades severas en la repetición de logatomos (pseudopalabras), palabras, frases, y en la denominación de objetos. Igualmente, se encontraron dificultades en la prueba de memoria verbal a corto y a largo plazo (aprendizaje), pero que se explican por las dificultades de lenguaje mencionadas anteriormente (repetición y denominación). Las praxias ideomotoras y orofonatorias se encontraban preservadas, mientras que se evidencian dificultades moderadas en las praxias visuoconstructivas.

En la evaluación posquirúrgica, HJ se encontraba alerta, orientado en persona y severamente desorientado en tiempo y lugar. Se encontró una buena articulación y prosodia del lenguaje, pero con una fluidez moderadamente afectada, con frecuentes parafasias fonológicas y, en menor medida, semánticas, y con dificultades severas en la repetición de logatomos (pseudopalabras), palabras, frases y en la denominación de objetos. Se obtuvieron desempeños por debajo de lo esperado en memoria a corto y a largo plazo, los cuales se explican por las dificultades de lenguaje mencionadas. No obstante, hay que resaltar que pudo evocar un mayor número de estímulos comparado con la valoración prequirúrgca. Las praxias ideomotoras y orofonatorias se encontraron preservadas, mientras que se seguían presentando dificultades moderadas en las praxias visuoconstructivas.

En conclusión, $\mathrm{HJ}$ presentaba como diagnóstico principal una afasia de conducción y adicionalmente una apraxia visuoconstructiva. Estas dificultades se encontraron en la evaluación prequirúrgica y posquirúrgica, aunque con mejores desempeños cuantitativos y cualitativos en esta última. 
TABLA 1

Resultados cuantitativos de las pruebas de evaluación neuropsicológica

\begin{tabular}{|c|c|c|c|c|}
\hline \multicolumn{2}{|c|}{ Función cognitiva evaluada } & Subtest aplicado & $\begin{array}{c}\text { Puntuaciones evaluación } \\
\text { prequirúrgica }\end{array}$ & $\begin{array}{c}\text { Puntuaciones evaluación } \\
\text { postquirúrgica }\end{array}$ \\
\hline \multirow{3}{*}{$\begin{array}{l}\text { Orienta- } \\
\text { ción }\end{array}$} & \multirow{3}{*}{$\begin{array}{l}\text { Orientación en } \\
\text { persona, lugar y } \\
\text { tiempo }\end{array}$} & Persona & $\begin{array}{l}\mathrm{PD}: 3 / 7 \\
\mathrm{PC}:<10\end{array}$ & $\begin{array}{l}\text { PD: } 4 / 7 \\
P C:<10\end{array}$ \\
\hline & & Tiempo & $\begin{array}{l}\text { PD: } 6 / 23 \\
P C:<10\end{array}$ & $\begin{array}{l}\mathrm{PD}: 6 / 23 \\
\mathrm{PC}:<10\end{array}$ \\
\hline & & Lugar & $\begin{array}{l}\mathrm{PD}: 0 / 5 \\
\mathrm{PC}:<10\end{array}$ & $\begin{array}{l}\text { PD: } 0 / 5 \\
\text { PC: }<10\end{array}$ \\
\hline \multirow{8}{*}{ Praxias } & \multirow{8}{*}{$\begin{array}{l}\text { Praxias ideomoto- } \\
\text { ras, visoconstruc- } \\
\text { tivas. }\end{array}$} & $\begin{array}{l}\text { Praxis constructiva gráfica a la } \\
\text { orden }\end{array}$ & $\begin{array}{l}\text { PD: } 0 / 8 \\
\text { PC: }<10\end{array}$ & $\begin{array}{l}\text { PD: } 3 / 8 \\
\text { PC: }<10\end{array}$ \\
\hline & & $\begin{array}{l}\text { Praxis constructiva gráfica a la } \\
\text { copia }\end{array}$ & $\begin{array}{l}\text { PD: } 12 / 18 \\
\text { PC: }<10\end{array}$ & $\begin{array}{l}\text { PD: } 5 / 18 \\
\text { PC: }<10\end{array}$ \\
\hline & & Imitación de posturas unilaterales & $\begin{array}{l}\text { PD (derecha): 10/10 } \\
\text { PD: (izquierda): 10/10 } \\
\text { PC (derecha): } 95 \\
\text { PC (izquierda): } 95\end{array}$ & $\begin{array}{l}\text { PD (derecha): 8/10 } \\
\text { PD (izquierda) }: 8 / 10 \\
\text { PC (derecha) }:<10 \\
\text { PC (izquierda) }:<10\end{array}$ \\
\hline & & $\begin{array}{l}\text { Gesto simbólico de comunicación } \\
\text { a la orden }\end{array}$ & $\begin{array}{l}\text { PD (derecha) }: 8 / 10 \\
\text { PD (izquierda) }: 8 / 10 \\
\text { PC (derecha) }:<10 \\
\text { PC (izquierda) }:<10\end{array}$ & $\begin{array}{l}\text { PD (derecha): 10/10 } \\
\text { PD (izquierda): 10/10 } \\
\text { PC (derecha): } 95 \\
\text { PC (izquierda): } 95\end{array}$ \\
\hline & & $\begin{array}{l}\text { Gesto simbólico de comunicación } \\
\text { a la imitación }\end{array}$ & $\begin{array}{l}\text { PD (derecha):9/10 } \\
\text { PD (izquierda):9/10 } \\
\text { PC (derecha): } 95 \\
\text { PC (izquierda): } 95\end{array}$ & $\begin{array}{l}\text { PD (derecha):10/10 } \\
\text { PD (izquierda): } 10 / 10 \\
\text { PC (derecha): } 95 \\
\text { PC (izquierda): } 95\end{array}$ \\
\hline & & $\begin{array}{l}\text { Mímica del uso de objetos a la } \\
\text { orden }\end{array}$ & $\begin{array}{l}\text { PD (derecha): 8/10 } \\
\text { PD (izquierda) }: 8 / 10 \\
\text { PC (derecha) }:<10 \\
\text { PC (izquierda) }:<10\end{array}$ & $\begin{array}{l}\text { PD (derecha): 4/10 } \\
\text { PD (izquierda): 4/10 } \\
\text { PC (derecha) }:<10 \\
\text { PC (izquierda) }:<10\end{array}$ \\
\hline & & $\begin{array}{l}\text { Mímica del uso de objetos a la } \\
\text { imitación }\end{array}$ & $\begin{array}{l}\text { PD (derecha): 9/10 } \\
\text { PD (izquierda): 9/10 } \\
\text { PC (derecha): } 30 \\
\text { PC (izquierda): } 30\end{array}$ & $\begin{array}{l}\text { PD (derecha): 9/10 } \\
\text { PD (izquierda): 9/10 } \\
\text { PC (derecha): } 30 \\
\text { PC (izquierda): } 30\end{array}$ \\
\hline & & Orofonatorias en imitación & $\begin{array}{l}\text { PD: } 16 / 20 \\
\text { PC: }<10\end{array}$ & $\begin{array}{l}\text { PD: } 18 / 20 \\
\text { PC: } 10\end{array}$ \\
\hline \multirow{2}{*}{$\begin{array}{l}\text { Memoria } \\
\text { Verbal }\end{array}$} & \multirow{2}{*}{ Memoria verbal } & $\begin{array}{l}\text { Memoria verbal de textos-Evoca- } \\
\text { ción inmediata }\end{array}$ & $\begin{array}{l}\mathrm{PD}: 2.5 / 23 \\
\mathrm{PC}:<10\end{array}$ & $\begin{array}{l}\text { PD: } 10.5 / 23 \\
\text { PC: }<10\end{array}$ \\
\hline & & $\begin{array}{l}\text { Memoria verbal de textos-Evoca- } \\
\text { ción diferida }\end{array}$ & $\begin{array}{l}\mathrm{PD}: 3.5 / 23 \\
\mathrm{PC}:<10\end{array}$ & $\begin{array}{l}\text { PD: } 5 / 23 \\
\text { PC: }<10\end{array}$ \\
\hline \multirow{3}{*}{ Lenguaje } & Fluidez verbal & $\begin{array}{l}\text { Fluencia verbal y contenido infor- } \\
\text { mativo. Descripción de la lámina }\end{array}$ & $\begin{array}{l}\text { PD: } 3 / 6 \\
\text { PC: }<10\end{array}$ & $\begin{array}{l}\text { PD: } 3 / 6 \\
P C:<10\end{array}$ \\
\hline & \multirow{2}{*}{ Prosodia } & Ritmo & $\begin{array}{l}\text { PD: } 3 / 3 \\
\text { PC: } 95\end{array}$ & $\begin{array}{l}\text { PD: } 3 / 3 \\
\text { PC: } 95\end{array}$ \\
\hline & & Melodía & $\begin{array}{l}\text { PD: } 3 / 3 \\
\text { PC: } 95\end{array}$ & $\begin{array}{l}\text { PD: } 3 / 3 \\
\text { PC: } 95\end{array}$ \\
\hline
\end{tabular}




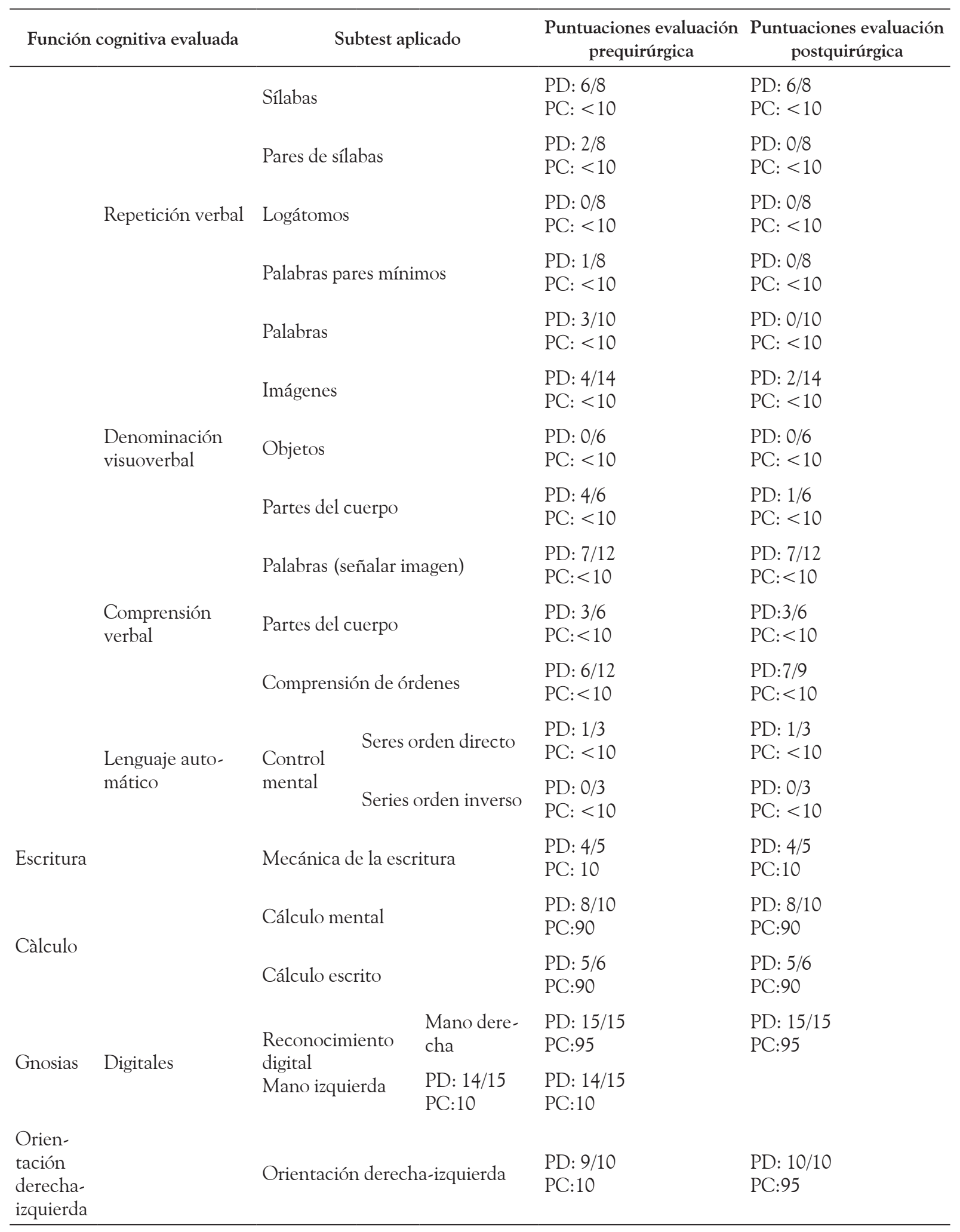

PD: puntuación directa; PC: Puntuación centil.

Fuente: elaboración propia. 


\section{Discusión}

Al realizarse la evaluación neuropsicológica de $\mathrm{HJ}$, se determinó que no existía un síndrome de Gerstmann, el cual se ha asociado a alteraciones parietales izquierdas (Lebrun, 2005) y por el contrario, se pudo confirmar que el diagnóstico correspondía a una afasia de conducción en la que se afecta severamente la capacidad para repetir y con relativa frecuencia la habilidad para denominar (Anderson et al., 1999).

A partir de la entrevista, se definió que esta alteración no era consecuencia de la primera cirugía realizada y que, por el contrario, surgió cuando el astrocitoma recidivó. Lo anterior muestra que la semiología neuropsicológica no depende exclusivamente de la topografía lesional, sino que entran en juego factores como el tipo de enfermedad neurológica, ya que los tumores son patologías más extensas que producen efectos distantes por la distorsión del cerebro, la tracción de los vasos sanguíneos y la obstrucción del sistema ventricular (Geschwind, 2009). Así mismo, cuando se analizó de una forma integrada el perfil neuropsicológico de $\mathrm{HJ}$ con las neuroimagenes estructurales (resonancia magnética cerebral simple y con contraste), se determinó que no existe una relación limitada exclusivamente al fascículo arqueado, sino que, por el contrario, existía un compromiso córticosubcortical que involucraba estructuras parietales posteriores superiores e inferiores, el lóbulo temporal posterior y el lóbulo occipital anterior. La extensión y localización de la lesión nos llevan a concluir que estos trastornos no tienen una relación exclusivamente topográfica y que, por el contrario, se debe plantear un análisis en términos de circuitos neurales implicados diferencialmente en procesos cognitivos, que dependiendo de procesos particulares de aprendizaje y de un sinnúmero de variables como lo son la escolarización, el género, la dominancia manual, etc., van a determinar las particularidades de las configuraciones neuronales de cada individuo, que llevan a que, en ciertas ocasiones, los procesos cognitivos estén asociados, y en otras circunstancias a que estén disociados. Lo anterior se concluye a partir de la valoración del pa- ciente, pues en él no se encontraron las asociaciones clásicas entre estructura, lateralización cerebral y función cognitiva. Esto nos lleva a la necesidad de reconceptualizar el abordaje de los pacientes, dejando de lado una concepción localizacionista y neofrenológica, por una conceptualización de redes neurales que muestran patrones corticales de actividad sincronizada locales y globales que integran la actividad sensoriomotora (Freeman, 2005) y que nos plantean síndromes particulares, ya que, a pesar de la extensión de la lesión, HJ no presenta agnosia visual (asociada a alteraciones occipitales), ni problemas en la comprensión del lenguaje (asociado tradicionalmente a áreas temporales posteriores, área de Wernicke), ni alexia y agrafia aisladas asociadas con alteraciones del giro angular (Kolb \& Wishaw, 2006), y por el contrario, presenta apraxia visuocontructiva que se ha asociado a alteraciones del hemisferio derecho y que en este caso no presenta anormalidades.

Incluso, tradicionalmente se ha relacionado el lenguaje como dependiente del hemisferio izquierdo, pero con los estudios de neuroimagen funcional, se ha obtenido información valiosa respecto al papel que cumple el hemisferio derecho - mal llamado no dominante- en la producción y comprensión del lenguaje, en el aprendizaje de una segunda lengua y en la recuperación del lenguaje después de una alteración cerebral (Ansaldo, Arguin \& Lecours, 2002; Sturm et al., 2004; Hull \& Vaid, 2007).

Adicionalmente, los estudios clínicos están ayudando a redefinir el estudio de las asimetrías cerebrales y las consecuencias de lesiones lateralizadas, pues se ha observado, en algunos pacientes, que a pesar de existir una lesión en un único hemisferio, se encuentran alteraciones neuropsicológicas que tradicionalmente se asociaban al hemisferio opuesto. Estos estudios señalan que el funcionamiento cerebral implica redes neurales bilaterales que trabajan simultáneamente y que se conectan a través de estructuras como el cuerpo calloso (Selnes \& Whitaker, 2006; Ihara et al., 2010). Lo anterior, se evidencia en HJ, debido a que si no existiera un funcionamiento en red que implicara la interacción de los dos hemisferios, no 
se encontrarían alteraciones visuocontructivas, que tradicionalmente se han asociado con alteraciones del hemisferio derecho, el cual en este paciente estaba sano.

¿Por qué no se presentan esas alteraciones y sí se mantiene la afasia de conducción? Una primera posibilidad es que por procesos de plasticidad cerebral se hubiera presentado una reconfiguración cortical, por lo cual se mantienen estas funciones preservadas (Pascual-Leone, Amedi, Fregni \& Merabet, 2005).

Otra explicación radica en que los procesos lingüísticos dependen de la interacción córticosubcortical-cortical, y diferentes investigaciones están señalando que las estructuras subcorticales no tienen el mismo potencial de reorganización que estructuras corticales (Duffau, 2009).

Por último, desde modelos cognitivos y poscognitivos, se ha debatido y se están replanteando las concepciones clásicas y lineales del lenguaje, en las que procesos como la repetición dependen primero de un input sensorial (auditivo), para luego conectarse con áreas de programación motora (área de Broca). En la actualidad y a partir de diferentes técnicas de neuroimagen funcional, se están desarrollando modelos más complejos que integran aspectos motores y sensoriales del lenguaje como un proceso bidireccional y en paralelo, en los que estructuras como el fascículo arqueado no solo transmiten información desde las áreas temporales a las frontales, sino también en la dirección opuesta (Schmahmann et al., 2007). En el mismo sentido, el estudio de las neuronas espejo ha abierto un amplio espectro de posibilidades, ya que muestran que estímulos sensoriales en tareas de imitación (repetición de estímulos auditivos y visuales) producen activación cerebral en áreas de programación motora, lo cual se puede relacionar con los procesos de desarrollo del habla en condiciones normales y que han revelado que el infante cuando escucha hablar, observa e imita los movimientos de la boca, repitiendo lo que le dicen, lo cual facilita simultáneamente la comprensión del lenguaje (Iacoboni, 2009; Peña-Casanova, 2007).

En conclusión, el caso estudiado contribuye a la redefinición de las afasias y en particular a la afasia de conducción, ya que los pacientes no presentan las mismas capacidades preservadas, ni las mismas alteraciones cognitivas, a pesar de tener lesiones cerebrales que comprometan las mismas estructuras. Se deben reconceptualizar los diferentes cuadros afásicos y analizarlos en un espectro más amplio que la base anatómica y dicotómica, que plantea que las afasias no fluentes se dan por lesiones anteriores y las afasias fluentes, como la afasia de conducción, por lesiones posteriores, ya que el fascículo arqueado parece estar implicado funcionalmente en la repetición verbal, pero su integridad y su funcionamiento no son indispensables para tal proceso cognitivo (Anderson et al., 1999; Bernal \& Ardila, 2009; Buchsbaum et al., 2011). Por otra parte, la literatura muestra, con mayor frecuencia, que los pacientes con afasia de conducción presentan otros síntomas asociados y que en $\mathrm{HJ}$ se manifiestan por las dificultades en la repetición de pseudopalabras, por dificultades en la denominación y por una apraxia visuocontructiva (Bernal \& Ardila, 2009). Lo anterior, nos lleva a seguir las líneas de investigación que plantean que no existe una correlación directa entre la topografía lesional y la semiología clínica neuropsicológica en lesiones posteriores, ya que se pueden encontrar procesos cognitivos asociados y disociados.

\section{Referencias}

Anderson, J., Gilmore, R., Roper, S., Crosson, B., Bauer, R. M., Nadeau, S. et al. (1999). Conduction aphasia and the arcuate fasciculus: A reexamination of the Wernicke-Geshwind model. Brain Eु Language, 70(1), 1-12.

Ansaldo, A. I., Arguin, M. \& Lecours, A. R. (2002). The contribution of the right cerebral hemisphere to the recovery from aphasia: A single longitudinal case study. Brain and Language, 82 (2), 206-222.

Baldo, J., Klostermann, E. \& Dronkers, N. (2008). It's either a cook or a baker: Patients with conduction aphasia get the gist but lose the trace. Brain 8 language, 105(2), 134-140.

Bartha, L., Mariën, P., Powe, W. \& Benke, T. (2004). Linguistic and neuropsychological deficits in crossed 
conduction aphasia. Report of three cases. Brain Ë Language, 88(1), 83-95.

Bernal, B. \& Ardila, A. (2009). The role of the arcuate fasciculus in conduction aphasia. Brain, 132, 2309-2316.

Buchsbaum, B., Baldo, J., Okada, K., Berman, K., Dronkers, N., D Ésposito, M. \& Hickok, G. (2011). Conduction aphasia, sensory-motor integration, and phonological short-term memory: An aggregate analysis of lesion and fMRI data. Brain $\mathcal{E}$ Language, 116. (doi:10.1016/j.bandl.2010.12.001)

Catani, M. \& Mesulam, M. (2008). The arcuate fasciculus and the disconnection theme in language and aphasia: History and current state. Cortex, 44(8), 953-961.

Duffau, H. (2009). Does post-lesional subcortical plasticity exist in the human brain? Neuroscience Research, 65(2), 131-135.

Freeman, W. (2005). A field-theoretic approach to understanding scale-free neocortical dynamics. Biological Cybernetics, 92 (6), 350-359.

Geschwind, N. (2009). Problems in the anatomical understanding of aphasias. En A. Benton (Ed.), Brain and Behavior: Research in Clinical Neuropsychology (pp. 107-128). Chicago: Aldine.

Hull, R. \& Vaid, J. (2007). Bilingual language lateralization: A meta-analytic tale of two hemispheres. Neuropsychologia, 45(9), 1987-2008.

Iacoboni, M. (2009). Imitation, empathy, and mirror neurons. Annual Review of Psychology, 60, 653-670.

Ihara, A., Hirata, M., Fujimaki. N., Goto, T., Umekawa, Y. Fujita, N. et al. (2010). Neuroimaging study on brain asymmetries in situs inversus totalis. Journal of the Neurological Sciences, 288(1-2), 72-78.

Kolb, B. \& Wishaw, I. (2006). Neuropsicología Humana ( $5^{\mathrm{a}}$ ed.). Madrid: Médica Panamericana.
Lebrun, Y. (2005). Gertmann's Syndrome. Journal of Neurolinguistics, 18, 317-326.

Matsumoto, R., Okada, T., Mikuni, N., Mitsueda-Ono, T., Taki, J., Sawamoto, N. et al. (2008). Hemispheric asymmetry of the arcuate fasciculus: A preliminary diffusion tensor tractography study in patients with unilateral language dominance defined by Wada test. Journal of Neurology, 255 (11), 1703-1711.

Pascual-Leone, A., Amedi, A., Fregni, F. \& Merabet, L. B. (2005). The Plastic Human Brain Cortex. Annual Reviews Neuroscience, 28, 377-401.

Peña-Casanova, J. (1990). Programa integrado de exploración neuropsicológica "Test Barcelona" [Manual]. Barcelona: Masson.

Peña-Casanova, J. (2007). Neurología de la Conducta y Neuropsicología. Madrid: Médica Panamericana.

Robin, D., Jacks, A., Hageman, C., Clark, H. \& Woodworth, G. (2008). Visuomotor tracking abilities of speakers with apraxia of speech or conduction aphasia. Brain Ė Language, 106(2), 98-106.

Schmahmann, J., Pandya, D., Wang, R., Dai, G., D'Arceuil, H., de Crespigny, A. \& Wedeen, V. (2007). Association fibre pathways of the brain: Parallel observations from diffusion spectrum imaging and Autoradiography. Brain, 130, 630-653.

Selnes, O. \& Whitaker, A. (2006). Anatomical asymmetries versus variability of language areas of the brain. En K. Brown (Ed.), Encyclopedia of Language $\mathcal{E}^{2}$ Linguistics (Vol. 1, pp. 240-244). London: Elsevier. Sturm, W., Longoni, F., Weis, S., Specht, K., Herzog, H., Vohn, R. et al. (2004). Functional reorganisation in patients with right hemisphere stroke after training of alertness: A longitudinal PET and AMRI study in eight cases. Neuropsychologia, $42(4), 434-450$. 
